\title{
Discrete vibro-tactile feedback prevents object slippage in hand prostheses more intuitively than other modalities
}

(C) 2018 IEEE. Personal use of this material is permitted. Permission from IEEE must be obtained for all other uses, in any current or future media, including reprinting/republishing this material for advertising or promotional purposes, creating new collective works, for resale or redistribution to servers or lists, or reuse of any copyrighted component of this work in other works. The DOI associated with this publication is 10.1109/TNSRE.2018.2851617. The published version is available at the following link:

https://ieeexplore.ieee.org/document/8400576/ 


\title{
Discrete vibro-tactile feedback prevents object slippage in hand prostheses more intuitively than other modalities
}

\author{
Mohamed Aboseria, Francesco Clemente, Leonard F. Engels, Christian Cipriani, Senior Member, \\ IEEE
}

\begin{abstract}
In the case of a hand amputation, the affected can use myoelectric prostheses to substitute the missing limb and regain motor functionality. Unfortunately, these prostheses do not restore sensory feedback, thus users are forced to rely on vision to avoid object slippage. This is cognitively taxing, as it requires continuous attention to the task. Thus, providing functionally effective sensory feedback is pivotal to reduce the occurrence of slip events and reduce the users' cognitive burden. However, only a few studies investigated which kind of feedback is the most effective for this purpose, mostly using unrealistic experimental scenarios. Here we attempt a more realistic simulation of involuntary hand opening and subsequent recovery of a stable grasp of the slipping object using a robotic hand operated by the subjects through a standard myoelectric control interface. We compared three stimulation modalities (vision, continuous grip force feedback and discrete slip feedback) and found that the discrete feedback allowed subjects to have higher success rates (close to $\mathbf{1 0 0} \%$ ) in terms of objects recovered from slippage, basically requiring no learning. These results suggest that this simple yet effective feedback can be used to reduce grasp failures in prosthetic users, increasing their confidence in the device.
\end{abstract}

Index Terms-Myoelectric prosthesis; prosthetic hand; sensory feedback; slip feedback; unexpected event.

\section{INTRODUCTION}

$\mathrm{S}$ ENSORIMOTOR control in humans is affected by delays caused by the limited conduction velocity of the neural signals between the brain and the periphery [1]. For this reason, stereotypical motor actions are primarily based on forward models inbuilt in the brain (motor repertoire), rather than on online sensory feedback mechanisms [2], [3]. During a motor action, our brain predicts the sensory consequences of such actions based on the forward model, and compares the expected and actual (online) sensory measurements, in order to issue feedback responses to the current action [1], [4] and to update the forward models for future ones [2]. In fact, in the case of an ideal model and with no external perturbations, the motor action would be perfectly executed and there would be no need for

This work was partially supported by the European Commission under the DeTOP project (GA \#687905), by the European Research Council under the MYKI project (ERC-2015-StG, Grant no. 679820) and by INAIL (Italian workers' compensation Authority) under the CECA2020 project (PPRAS 1/2). MA was partially supported by a Whitaker International Fellowship awarded by the Institute of International Education. online sensory measurements [2]. However, (i) the inaccuracy of the central planning (i.e. wrong brain predictions) and (ii) stochastic noise in peripheral execution of a movement generate motor noise leading to differences between the intended and the actual motor output [3], [5], and in turn between the expected and actual sensory measurements.

An upper limb amputation severely impairs the motor and sensory system of the subject, invalidating his/her motor repertoire. The motor functions may be partially recovered by a myoelectric prosthesis, i.e. an electromechanical device controlled by the electromyographic (EMG) signals of the residual limb [6]. In this case, the prosthesis becomes an active part of the motor chain and of the forward model. Although the EMG provides information on the user's intention, as it is typically recorded from the skin by surface electrodes, there are technical difficulties in acquiring reliable EMG signals. Said signals can be affected by many factors, including neighbouring muscle activity, unreliable electrode positioning, sweating, and distortion due to the signal traveling through different subcutaneous tissues [7]. In addition, since the electrodes are housed inside the prosthetic socket, motion artefacts can be generated due to relative movements between electrodes and skin. All of these noise sources can result in unintentional control signals sent to the prosthesis, causing its activation (opening or closing) even in the absence of voluntary muscle contractions [8]. In other words, since the noise affecting EMG control is very large, some kind of sensory feedback becomes crucial in order to compensate for unpredictable actions. For example, myoelectric hand users heavily (continuously) rely on visual and/or auditory feedback in order to ensure that the object does not slip from the grasping hand. This cognitive burden is one of the causes for the abandonment of myoelectric hands [9].

To tackle this cognitive burden while preventing slippage, several researchers have proposed automatic grip controllers based on the interaction forces with the grasped object [10], [11]. Similar mechanisms were implemented in clinical prostheses (e.g. SensorHand Speed, Otto Bock, Austria). Yet,

M. A. is with The BioRobotics Institute, Scuola Superiore Sant'Anna, Viale R. Piaggio 32, 56025 Pontedera (PI), Italy and the College of Medicine, SUNY Downstate Medical Center, Brooklyn (NY), USA (email: aboseria7@gmail.com)

F. C., L. F. E. and C. C. are with The BioRobotics Institute, Scuola Superiore Sant'Anna, Viale R. Piaggio 32, 56025 Pontedera (PI), Italy

(e-mails: \{f.clemente / 1.engels / ch.cipriani\}@santannapisa.it). 
in the case that an EMG signal is generated unintentionally, the slip control system cannot discern it from an intentional signal, and thus the hand could open (or close), causing the grasped object to drop (or crush, if fragile). In these cases, some level of cognitive burden cannot be avoided as explicit sensory feedback, with the users in-the-loop, becomes the only way to allow them to discern unintentional hand openings from intentional ones and trigger corrective actions before the drop occurs [2].

Explicit (supplementary) sensory feedback can be provided invasively or non-invasively, continuously or in a time-discrete fashion [12]. Invasive feedback can be provided using surgically implanted electrodes that electrically stimulate afferent nerves within the residual limb. In contrast, noninvasive feedback is based on the mechanical or electrical stimulation of the skin [12]. Notably, a few studies also specifically investigated sensory feedback for preventing object slippage [13]-[16]. However, they all employed virtual environments [14] and unrealistic control interfaces or slip conditions [13], [15], [16].

Traditionally, researchers have implemented systems that provided sensory feedback in a continuous fashion. Timediscrete feedback is an alternative to this approach. One particular way of providing discrete feedback is based on the Discrete Event-driven Sensory Feedback Control (DESC) policy, pioneered by Johansson and colleagues [1], [17]. This policy posits that manipulation tasks are organized by means of multi-modally encoded discrete sensory events, e.g. resulting from object contact, lift-off, etc. The nervous system monitors such events and uses them to apply control signals and, if necessary, to initiate corrective actions that are appropriate for the task and the current phase. Based on this framework, recent studies demonstrated that this type of feedback is readily integrated by healthy participants in their sensorimotor control [18]. In addition, delivering discrete vibro-tactile feedback at contact and release of an object led to significant improvements in grasp control in amputees using myoelectric prostheses [19]. Slip is signalled to the nervous system through the same receptors (rapidly adapting receptors 1 and 2) and in much the same manner: via single spikes or short bursts of nervous activity that cease during longer slips [20]. Consequently, it seems sensible to also relay information about slip discretely, extending the DESC principles.

Accordingly, in this study, we aimed at investigating the potential benefit of both continuous and discrete sensory feedback strategies to prevent object slippage, in a realistic scenario. The investigated strategies were continuous mechanotactile feedback (a pressure proportional to the grip force applied on the object) and discrete vibro-tactile feedback (notifying the user if there was a subtle decrease in grip force). The task required healthy subjects to react to a sudden and unpredictable opening of a robotic hand, while holding an object, by controlling it using a conventional EMG controller. The subjects had to reclose the hand before the object dropped, while avoiding to virtually break it upon re-grasping. We compared subjects' performance (reaction time, success rate and percentage of slipped and crushed objects) with tactile feedback and with visual feedback only, with and without a supplementary cognitive task. The experiment was repeated for three consecutive days in order to investigate the integration of the different feedback modalities with time.

Building on our previous work [18], [19], we hypothesized that the simpler discrete feedback would be easier to integrate into one's sensorimotor control allowing for better initial performance. Yet, in the long run, as it provides specific grip force information, we expected that the continuous pressure feedback would allow the users to better modulate the EMG response (and thus the grip force exerted on the object) [21]. We predicted that this would have resulted in less broken objects, as shown in previous studies [22], [23]. Results proved that users could indeed take advantage of both feedback modalities, and that both were more efficient in preventing object slippage than vision alone. Yet, even after considerable training (i.e. on the third day), the discrete vibro-tactile feedback outperformed the continuous mechano-tactile feedback as measured by all the performance metrics. This outcome confirms that, if useful at all, the more complex continuous pressure information could provide functional benefits only after additional training, and it encourages further investigation into clever and simple sensory feedback schemes to effectively improve the ability of upper limb amputees in controlling their myoelectric prosthesis.

\section{MATERIALS AND METHODS}

\section{A. Subjects}

Ten healthy, unimpaired subjects participated in the study (age 22-31, six females, all right-handed). Written informed consent in accordance with the Declaration of Helsinki was obtained from each subject before conducting the experiments. This study was approved by the local ethical committee of the Scuola Superiore Sant'Anna, Pisa, Italy (ref. no. 02/2017).

\section{B. Materials}

The experimental platform (Fig. 1) consisted of a robotic hand with force sensors embedded in the digits, a test object, a PC running a custom application, two EMG electrodes and a sensory feedback system comprising a tactor and a miniature vibrator.

The robotic hand was a right-handed version of the IH2 Azzurra hand (Prensilia SRL, Italy). The hand was fixed on a stand on a table, and movements were limited to only flexionextension of the thumb and index finger. Embedded within those two digits were force sensors to measure the grip force (GF, defined as the average of the two readings) applied while grasping the test object (a $6 \times 6 \times 13 \mathrm{~cm}^{3}$ box weighing $70 \mathrm{~g}$ ). The object did not deform during grasping. The minimum GF $\left(\mathrm{GF}_{\mathrm{MIN}}\right)$ required to stably hold the test object with the robotic hand was $0.4 \mathrm{~N}$.

A custom application (LabWindows/CVI, National Instruments, USA) was used to control the sensory feedback system and the robotic hand, as well as to record the GF and EMG signals through a data acquisition board (USB-6002, National Instruments, USA) at $100 \mathrm{~Hz}$. A graphical user 


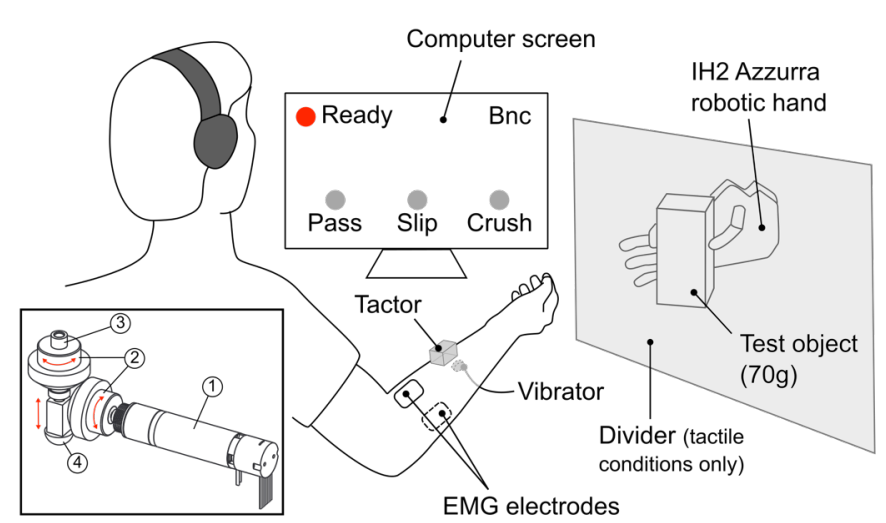

Fig. 1. Experimental setup. The subjects sat in front of a computer screen, and controlled a robotic hand via two EMG electrodes on the forearm. A tactor provided sensory feedback of the grasping force (pressure feedback condition), a miniature vibrator provided discrete feedback about the opening of the hand (vibration burst feedback condition, Bnc - see text). During these conditions, vision of the hand was obstructed by a divider. Throughout the experiment, the subjects wore noise-cancelling headphones that played white noise to prevent unintentional auditory feedback from the hand or feedback devices. The headphones further played a string of numbers during the cognitive task condition (see text). Inset: an overview of the tactor transmission. (1) DC motor, (2) bevel gears, (3) threaded screw and (4) output plunger. The red arrows depict the motion transmission from the electrical motor (1) to the output plunger (4).

interface (GUI) was displayed on a screen showing the outcome of the current trial (i.e. object slipped, object saved, object crushed) and allowing subjects to start a new trial. During the trials the GUI did not provide any additional information (i.e. it was static), to avoid any distraction or additional cognitive load on the user, especially when they had to rely on vision to complete the task (see below).

The signals acquired by the two EMG electrodes (13E125=50, Otto Bock HealthCare GmbH, Austria) opened/closed the hand at different speeds depending on the sign and magnitude of the difference between the signals, implementing the well-known two-state amplitude modulated controller [24] akin to clinical prostheses.

The sensory feedback system was a custom-built device that included a tactor and a miniature vibrator. The tactor $(78 \times 33$ $\times 24 \mathrm{~mm}$ ) comprised a DC motor and a transmission that allowed to transform a rotary motion of the motor into a linear motion of a plunger ( $7 \mathrm{~mm}$ stroke), used to press on the skin (Fig. 1). The miniature vibrator $(3.4 \mathrm{~mm}$ thickness $\times 12 \mathrm{~mm}$ diameter) could vibrate at a frequency of $150 \mathrm{~Hz}$ with a peakto-peak force amplitude of about 0.32 N (Pico Vibe 312-101, Precision Microdrives, UK). The position of the plunger and the duration of the vibration were driven by a custom microcontroller board by receiving commands from the host PC over a serial bus.

\section{Experimental protocol}

The experiment aimed at emulating the scenario of an unwanted opening of the prosthesis due to EMG control signals involuntarily generated by the user. During the experiment, the participant sat comfortably in front of a table with the dominant (i.e. the right) arm in a supine position on a cushion placed on the table. First, the electrodes were placed on the flexor carpi radialis and its antagonist muscles on the forearm of the subject.
Then, the tactor and the vibrator were placed midway between the elbow and the wrist, ventrally (Fig. 1). To prevent the subjects from hearing the motor noise of the hand, tactor or vibrator, they wore noise-cancelling ear muffs, which played white noise throughout the experiment.

Subjects were instructed to control the robotic hand using the EMG controller in order to save the object from falling by regrasping it as quickly as possible. The subjects were warned to treat the object as a fragile object that would break if grasped with an excessive grip force (see below).

For each experimental trial, the object was securely fixed in the hand (by the experimenter) using a pinch grip ( $\mathrm{GF}=1.4 \pm$ $0.1 \mathrm{~N}$ ). The subjects then initiated the trial by clicking on a button of the GUI and gained control of the robotic hand. At this point, the hand began to open randomly from 3 to 10 seconds after the trial started, in order to emulate an involuntary control signal and induce slippage. The opening speed of the digits was randomized (and balanced) over all trials between two levels $(10 \% \mathrm{~s}$ and $20 \% \mathrm{~s})$ in order to simulate involuntary control signals of different amplitudes. Because the opening of the hand was automatic throughout the whole experiment, subjects were only required to close the hand to re-grasp the object.

If the subjects failed to re-grasp the object in time, i.e. before it slipped off (within around 400-500 ms), the trial was considered as failed. If the subject re-grasped the object with an excessive GF (i.e. greater than $1.8 \mathrm{~N}-\mathrm{GF}_{\mathrm{MAX}}$ ), the object was considered "crushed" and the trial failed as well. If the subject reacted before the opening signal, the trial was stopped and repeated. All other trials were considered successful.

Trials differed in terms of the sensory feedback modality available to the subjects and if a cognitive task was present or not during the trial. The three feedback modalities tested were vision ( $\mathrm{V}$ condition), pressure ( $\mathrm{P}$ condition), and vibration burst (B condition). During the $\mathrm{V}$ condition, subjects had full view of the robotic hand and the object during slippage, mimicking the clinical situation of an amputee using his own prosthesis. During the $\mathrm{P}$ condition, the position of the tactor plunger was linearly mapped to the GF recorded by the robotic hand, in such a way that $7 \mathrm{~mm}$ of stroke corresponded to $2.5 \mathrm{~N}$ of GF and that a $0.7 \mathrm{~mm}$ in plunger displacement corresponded to $0.25 \mathrm{~N}$ of GF (10 steps discretization). In the B condition, a discrete, 100 $\mathrm{ms}$ vibration burst was delivered to the subjects when a decrease of $0.25 \mathrm{~N}$ in GF was detected by the sensors. The view of the hand was obstructed during both $\mathrm{P}$ and $\mathrm{B}$ conditions.

In half of the trials, the subjects were taxed with a supplementary cognitive task: in addition to the white noise, the earmuffs played randomly generated strings of single-digit numbers (0-9) at $1 \mathrm{~Hz}$. The subjects were asked to add one to each number and repeat it out aloud, so that the experimenter could check if they were paying attention to the task. The three feedback modalities and the cognitive task resulted in six test conditions for each subject: visual feedback with and without cognitive task (Vc, Vnc), continuous pressure feedback with and without cognitive task (Pc, Pnc), and discrete vibro-tactile feedback with and without cognitive task (Bc, Bnc).

Each condition included a series of 12 trials, after which a 


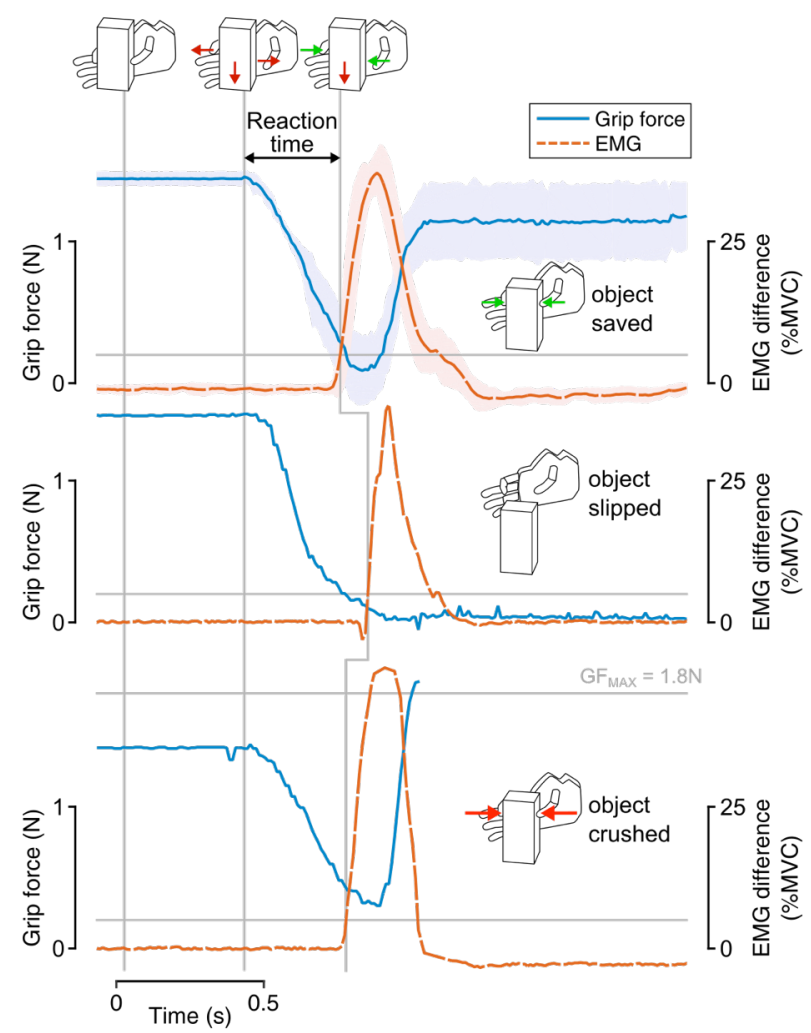

Fig. 2. Representative outcomes. Grip force and EMG difference time series (Bc condition, single subject). Above: successful trial (average time series among 12 trials). As the force decreased, the subject used the (vibro-tactile) feedback and increased his EMG signal to close the hand, thus saving the object from dropping. Middle: the object slipped from the grasp. Below: the object was virtually crushed.

new condition was tested, for a total of 72 trials. In order to evaluate mid-term learning effects, the experiment was repeated for three consecutive days, for a total of 216 trials per subject ( 72 trials/day $\times 3$ days). The conditions order was randomized both across days and subjects. On each day, subjects were given a few (three to five) random practice trials for each of the sensory feedback modalities to get them acquainted with the different closed-loop control schemes.

\section{Data analysis}

The experiment was assessed by means of the following performance metrics: percentage of successful trials (success rate), percentage of slipped or crushed objects (i.e. slip or crush rate), reaction time and variability of the reaction time. The reaction time was defined as the time delay between the beginning of the hand opening and the onset of the re-grasping signal from the EMG controller. The onset of re-grasp was experimentally defined as the time instant when the difference between EMG sensors (offline filtered with a 5-samples moving average filter) passed a fixed threshold (experimentally set to $5 \%$ maximum voluntary contraction, Fig. 2). The reaction time variability, defined as its standard deviation, provided information on the consistency of the motor performance among different trials.

Spearman's rank correlations $\left(\mathrm{r}_{\mathrm{s}}\right)$ were calculated to evaluate monotonic trends in the performance metrics across days for each test condition separately. The Kolmogorov-Smirnov test was used to verify that the data was normally distributed. If so, the effects of three factors ( 3 feedback strategies $\times 2$ presence of cognitive task $\times 3$ days) on all the performance metrics was evaluated using a multi-way analysis of variance ( $\mathrm{N}$-way ANOVA). Statistical significance in the N-way ANOVA was followed by post-hoc pairwise comparisons applying a TukeyKramer correction. In all cases, a p-value $<0.05$ was considered statistically significant.

\section{RESULTS}

Subjects were able to use the supplementary sensory feedback provided to react to the object slipping from the grasp (Fig. 2). In particular, the subjects used the sensory feedback stimuli to detect a decrease in GF, and to control the EMG signal to close the hand. If the reaction was fast enough, the control command yielded an increase in GF to adequate levels for the object to be saved from falling (Fig. 2, top). In the case the subject's reaction was too slow, the object slipped from the grasp (Fig. 2, middle). Finally, If the EMG activity was too strong, the GF passed the $\mathrm{GF}_{\mathrm{MAX}}$ threshold, and the object was virtually crushed (Fig. 2, bottom).

\section{A. Success rate}

The vibro-tactile feedback (Bnc) yielded median success rates close to $100 \%$, since the very first day; in all other conditions, the success rate was generally lower, improving with days/practice (Fig. 3). All of these trends proved significant (Pc $p=0.0440, \quad B c p<0.001, \quad V \quad p=0.0089, \quad P$ $\mathrm{p}=0.0484$ ), with the only exception of $\mathrm{Vc}$, showing a trend slightly above the significance threshold $(p=0.0747)$. The improvement between day 1 and day 3 was more relevant in the presence of the cognitive task, however the success rate on the third day never exceeded the performance observed without the cognitive task, for each of the three feedback modalities (this was true for all the performance metrics - cf. next paragraphs).

The ANOVA demonstrated that the sensory feedback modality had an effect on the subjects' success rate $(\mathrm{F}(2$, $165)=6.28, \mathrm{p}=0.023$ ). In particular, the post-hoc comparison proved that visual feedback ( $\mathrm{V}$ conditions) yielded worse performance than continuous pressure feedback ( $\mathrm{P}$ conditions, $\mathrm{p}=0.0287$ ) and discrete vibro-tactile feedback ( $\mathrm{B}$ conditions, $\mathrm{p}=0.0019$ ). $\mathrm{B}$ yielded a median success rate larger than $\mathrm{P}$, although this difference was not statistically relevant $(p=0.6709)$. The performance improved in consecutive days $(F(2,165)=22.18, p<0.001)$ in a highly significant manner from day 1 to day $2(\mathrm{p}<0.001)$, and not significantly from day 2 to day $3(\mathrm{p}=0.5363)$. The cognitive task had an effect on success rate as well $(\mathrm{F}(1,165)=93.04, \mathrm{p}<0.001)$, as expected.

\section{B. Reaction time}

As a trend, the Bnc condition yielded faster median reaction times than the other conditions for all days, being on average $355 \mathrm{~ms}$, and the outcomes were stable across the three days. All 


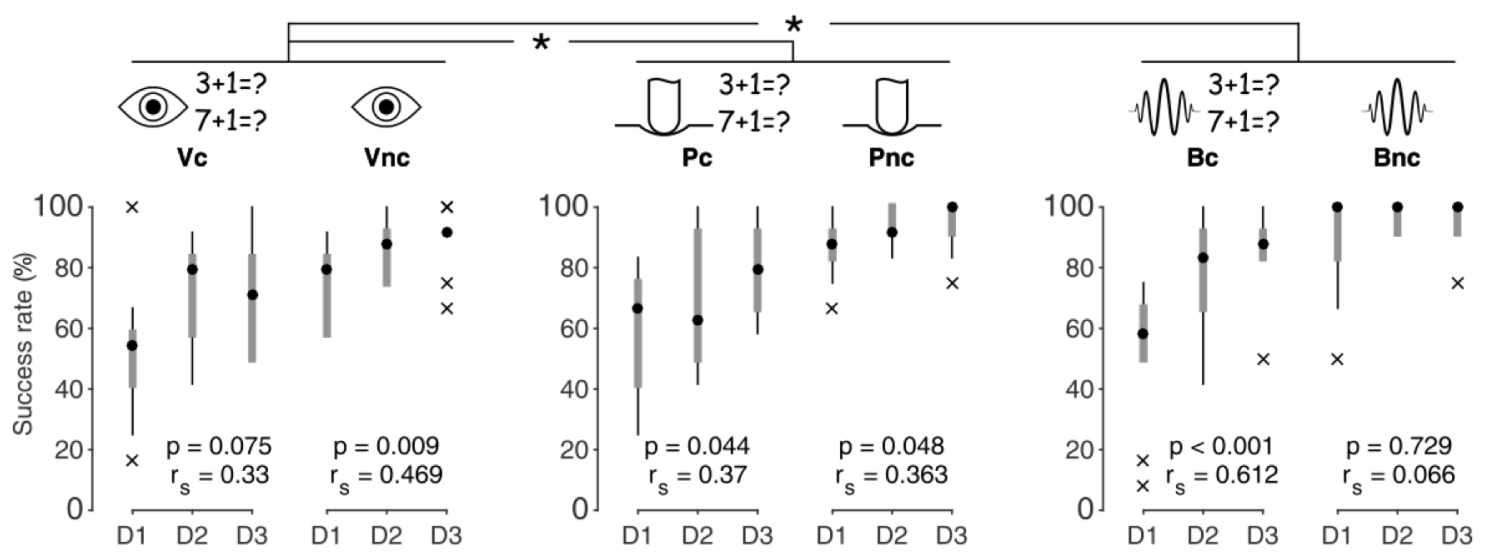

Fig. 3. Success rate. Success rates for each of the six conditions for the three consecutive days. Black dots indicate the median, grey boxes indicate the $25^{\text {th }}$ and $75^{\text {th }}$ percentile, and whiskers denote the most extreme data points excluding outliers (marked by " $\times$ "). * indicates $p<0.05$. $r_{s}$ and $p$ indicate the Spearman's rank correlation coefficients and related $\mathrm{p}$-value between the displayed variables.
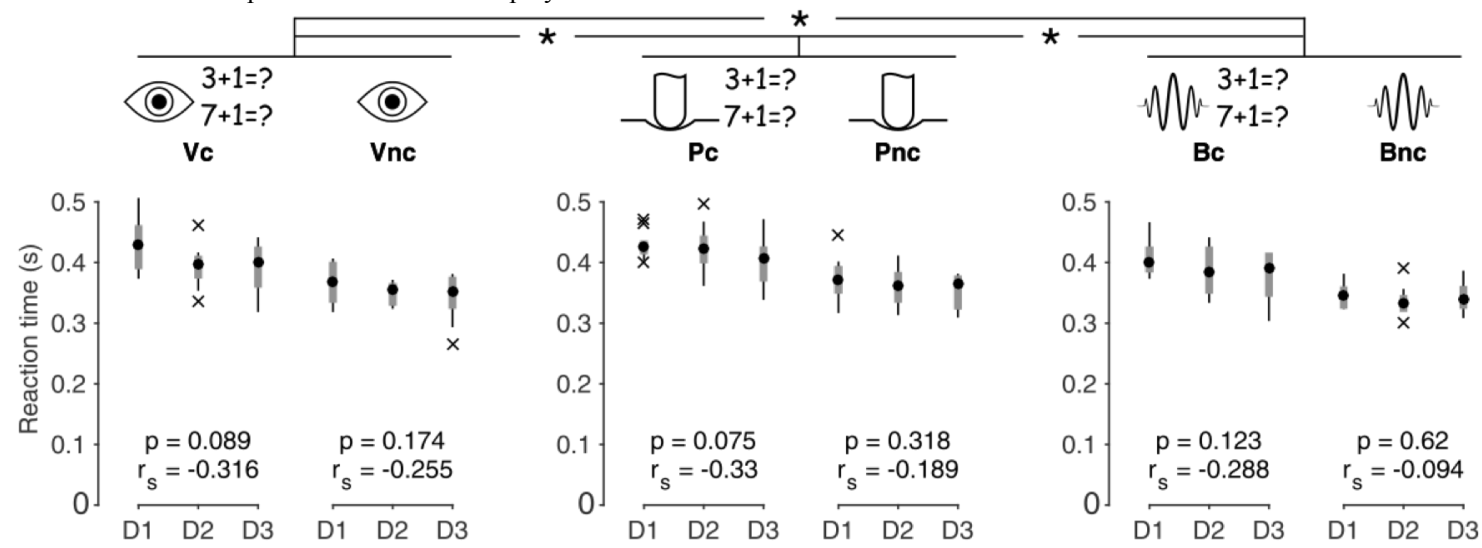

Fig. 4. Reaction time. Reaction time for each of the six conditions for the three consecutive days. Black dots indicate the median, grey boxes indicate the $25^{\text {th }}$ and $75^{\text {th }}$ percentile, and whiskers denote the most extreme data points excluding outliers (marked by “ $\times$ "). * indicates $p<0.05$. $r_{s}$ and $p$ indicate the Spearman's rank correlation coefficients and related p-value between the displayed variables.

other conditions showed negative trends, suggesting that subjects learned to react faster with practice, yet none of these trends proved statistically significant (Fig. 4).

The aggregated data demonstrated an effect of the feedback modality on the reaction time $(\mathrm{F}(2,165)=17.09, \mathrm{p}<0.001)$. Subjects had the fastest reaction times when receiving vibrotactile feedback, followed by vision, followed by pressure feedback (V vs. $\mathrm{B} p=0.0022$, V vs. $\mathrm{P} p=0.0376, \mathrm{P}$ vs. $\mathrm{B}$ $\mathrm{p}<0.001)$. However, the difference in reaction times was very small and did not influence the success rate significantly: although the subjects were faster in the $\mathrm{V}$ condition than in $\mathrm{P}$, their success rates proved better in the latter case (Fig. 3). As with the success rate, there was a significant effect of day on reaction time $(\mathrm{F}(2,165)=18.08, \mathrm{p}<0.001)$. The reduction was significant from day 1 to day $2(\mathrm{p}<0.001)$, but not between day 2 and day $3(\mathrm{p}=0.2737)$. Unsurprisingly, the presence of the cognitive task led to significantly longer reaction times $(\mathrm{F}(1$, $165)=247.77, \mathrm{p}<0.001)$.

Although generally decreasing, the variability of the reaction time (i.e. the standard deviation) did not change significantly across days in any of the conditions (Fig. 5), with the exception for the Pc condition $\left(r_{s}=-0.458, p=0.011\right)$.

The ANOVA demonstrated that the feedback modality had an effect on the reaction time variability $(F(2,165)=20.23$, $\mathrm{p}<0.001)$. The lowest variability was achieved when receiving vibro-tactile feedback, followed by pressure and vision (V vs. $\mathrm{B} \mathrm{p}<0.001, \mathrm{~V}$ vs. $\mathrm{P} \mathrm{p}=0.66, \mathrm{P}$ vs. $\mathrm{B} \mathrm{p}<0.001)$. There was a significant effect of days as well $(F(2,165)=6.43, p=0.002)$, although the only significant reduction was from day 1 to day 3 $(p=0.001)$. The cognitive task had an effect and led to significantly more variable reaction times as well $(\mathrm{F}(1$, $165)=14.99, \mathrm{p}<0.001)$.

\section{Crush rate}

The Bnc condition yielded zero median crush rates from the first day, akin to the success rate; in all other conditions, the crush rate was generally higher, decreasing significantly with practice/days (Fig. 6).

The crush rate was affected by the feedback strategy $(\mathrm{F}(2$, $165)=7.33, \mathrm{p}<0.001$ ), however the post hoc revealed a significant difference between the $\mathrm{V}$ and $\mathrm{B}$ conditions only ( $\mathrm{B}$ better, $\mathrm{p}<0.001)$. As with all the previous metrics, subjects lowered their crush rate significantly with days $(\mathrm{F}(2,165)=27.4$, $\mathrm{p}<0.001)$; this was significant from day 1 to day $2(\mathrm{p}<0.001)$ but not from day 2 to day $3(\mathrm{p}=0.1946)$. Additionally, as expected, the cognitive task significantly increased the crush rate in all conditions $(\mathrm{F}(1,165)=80.47, \mathrm{p}<0.001)$.

\section{Slip rate}

A very low slip rate was found in all the tested conditions from the first day onwards (always lower than $10 \%$, for all 


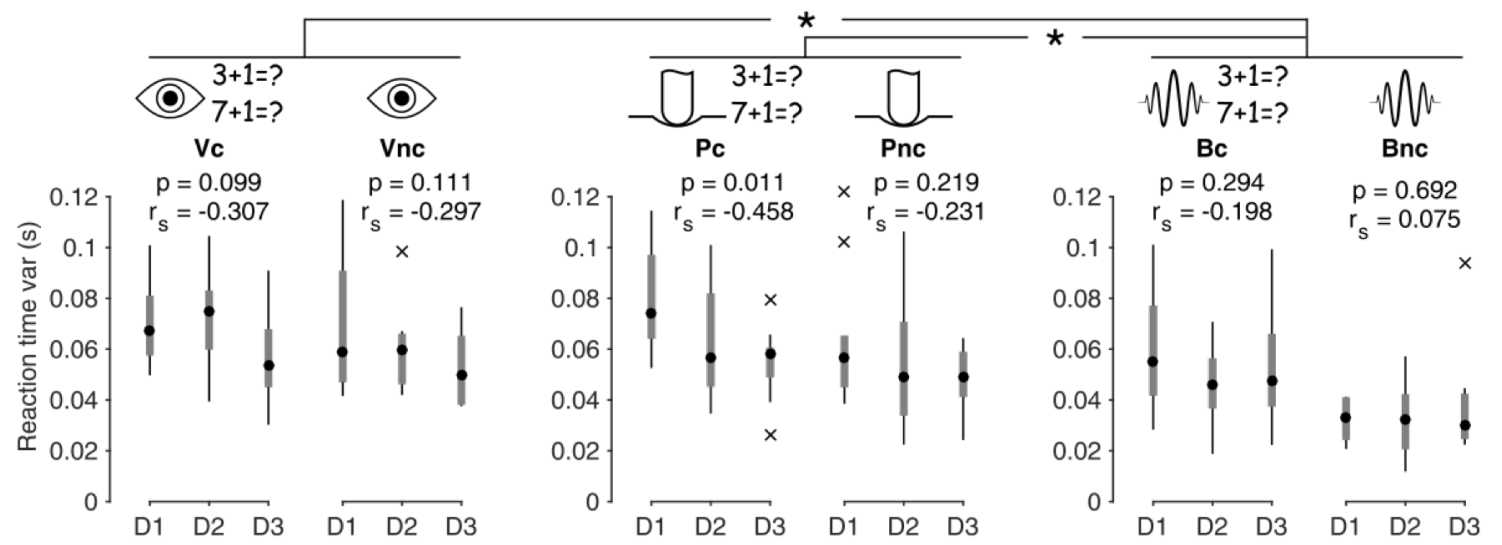

Fig. 5. Reaction time variability. Reaction time variability for each of the six conditions for the three consecutive days. Black dots indicate the median, grey boxes indicate the $25^{\text {th }}$ and $75^{\text {th }}$ percentile, and whiskers denote the most extreme data points excluding outliers (marked by " $\times$ "). * indicates $p<0.05$. $r_{s}$ and $p$ indicate the Spearman's rank correlation coefficients and related p-value between the displayed variables.

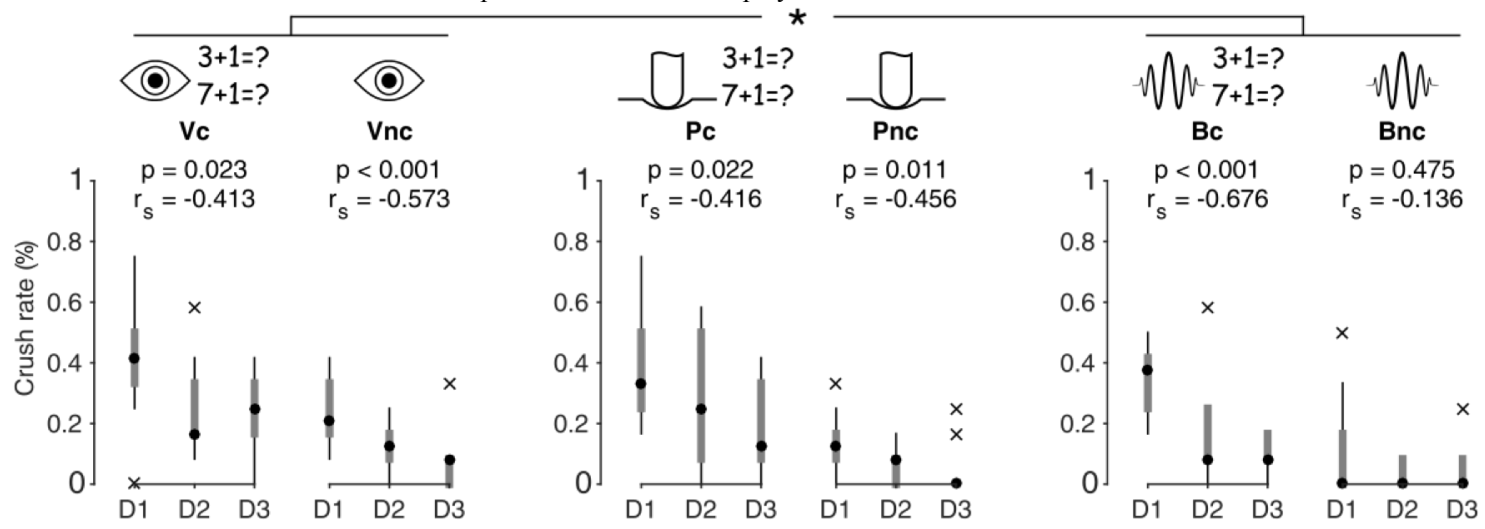

Fig. 6. Crush rate. Crush rate for each of the six conditions for the three consecutive days. Black dots indicate the median, grey boxes indicate the $25^{\text {th }}$ and $75^{\text {th }}$ percentile, and whiskers denote the most extreme data points excluding outliers (marked by “ $\times$ ”). * indicates $p<0.05$. $r_{s}$ and $p$ indicate the Spearman's rank correlation coefficients and related $\mathrm{p}$-value between the displayed variables.

conditions and all days). Thus, no significant trend was observed in the data. The result was confirmed by the ANOVA, which did not report any significant effect, of both the test condition $(\mathrm{F}(2,165)=2.26, \quad \mathrm{p}=0.1073)$ and the day $(\mathrm{F}(2,165)=0.58, \mathrm{p}=0.5603)$. The cognitive task was the only factor that proved significant, increasing the slip rate when present $(F(1,165)=13.4, \mathrm{p}<0.001)$.

\section{DISCUSSION}

In this work, we tested non-amputee volunteers by measuring the success rate, reaction time and the percentage of crushed and slipped objects during their attempts to recover a stable grasp of an object after randomized opening of the robotic hand that was holding it. While depriving subjects from visual feedback of the object, we partially replaced the biological afferent flow with artificial tactile stimuli pertaining to grip force ( $\mathrm{P}$ condition) or slip event (B condition). We then compared the subjects' performance with the case where only vision was available ( $\mathrm{V}$ condition). The presence of a cognitive task allowed us to mimic more realistic settings.

The study demonstrated that the success rate was significantly higher when tactile feedback ( $\mathrm{P}$ or $\mathrm{B}$ ) was provided to the participants. Indeed, in these conditions, subjects were able to reach $100 \%$ success rates within the timeframe of the study. In addition, with respect to continuous pressure feedback, the discrete vibro-tactile feedback enabled shorter and less variable reaction times (Fig. 4 and 5) and basically required no learning (i.e. it yielded median success rates close to $100 \%$ from day 1, Fig. 3). This is in line with our previous study demonstrating that humans can readily integrate discrete vibro-tactile feedback in their sensorimotor control of a hand prosthesis [18]. More specifically, these findings corroborate the DESC model, which hypothesizes that the central nervous system monitors specific peripheral sensory events marking the transitions between phases of the manipulative task and uses these events to apply control signals that are appropriate for the task and the current phase [4]. In this particular case, subjects used the sensory information on the slip event to initiate a corrective action and recover the object from the slip. These results suggest that amputees could rely on (discrete) tactile feedback to avoid object slippage, without needing to constantly look at the prosthesis while grasping.

The trained performance in the $\mathrm{P}$ condition was half-way between the $\mathrm{V}$ and $\mathrm{B}$ conditions. This contrasts with our prediction that the specific grip force feedback would have allowed subjects to regulate their motor action more finely (and thus the generated EMG signals), reducing the crush rate with respect to both the other conditions. This could be explained by a number of factors, including: skin sensitivity, cognitive effort, short training and maybe discrepancies between the experimental task complexity and the richness of the sensory feedback. First, the receptor density in the forearm (hence 
sensitivity, among others) is reduced compared to the hand/fingertips [25]. Second, the cognitive effort required to interpret continuous GF feedback should be considered. Indeed, even when accidentally severed and re-sutured nerves significantly re-innervate biological sensors - which should be vastly superior to any artificial sensory feedback-functional (sensory) recovery is unsatisfactory. The recovery significantly improves if the patient is in the early teens or younger [26] or if neural plasticity is favoured [27]. This suggests that, even under 'ideal' conditions, the limiting factor in sensory relearning is the patients' ability to reinterpret sensory information [28]. In other words, providing richer information to the user does not ensure improved sensory recovery. Third, it cannot be excluded that the training received by the subjects over three days was not sufficient to make them incorporate the additional feedback information in their internal model of the task. It thus remains to be tested if a longer training period would have shown different results. Finally, the task performed by the subjects was actually relatively simple (responding to a sensory stimulus with a single-degree-of-freedom motor action). For such simple tasks, easy-to-interpret and infrequent information is usually more effective than continuous feedback [29]. The simplicity of the task is also confirmed by the fact that all performance stagnated after two days of practice (no statistical difference was found between day two and three), whereas learning of complex motor tasks can take more than 20,000 trials before improvement stagnates [30]. Thus, a more complex task could be required to reveal the real benefits of such a feedback strategy.

As a difference between the two conditions, it should be noted that the continuous GF feedback was delivered through pressure, while the discrete feedback was delivered through vibration. In addition, the former started from a positive offset value (i.e. the stimulus decreased in time instead of increasing) and was discretized in 10 steps. However, the steps were dimensioned on the just noticeable difference for tactile stimuli (i.e. around $10 \%$ [31]), in order to ensure that the very first step resulted in a perceivable stimulus. This step was mapped to a very small GF difference $(0.25 \mathrm{~N})$, making the sensory feedback device very sensitive to GF variations. Nonetheless, a simple supra-threshold vibration proved more effective than a pressure feedback. This is interesting per se, scientifically, but it is also very relevant to the clinical situation. Indeed, any non-invasive sensory feedback device would likely be applied on skin areas with low receptor density. To exploit the richer information provided by a haptic system, a more sensitive skin site (e.g. the lips or the toes) or a longer training period should be investigated for conveying GF information.

Our results agree with previous studies. For example, the number of crushed objects was significantly lower in the B condition if compared to $\mathrm{V}$, akin to previous research [15]. The difference in reaction time between the $\mathrm{B}$ and $\mathrm{V}$ condition (on average $20 \mathrm{~ms}$ ) are in line with the literature reporting a faster reaction time for tactile stimuli with respect to visual stimuli [32]. More interestingly, the reduction in the variability of the reaction time in the $\mathrm{B}$ condition with respect to the other two conditions aligns with previous research [21] that suggests that the efficiency of vision or pressure feedback for predictive movement planning is lower than the one provided by feedback specifically designed for slip prevention. In the B condition, subjects received vibro-tactile stimuli containing only temporal information about the slip event. This is actually similar to what happens during normal grasping [20], which arguably allowed subjects to execute the task in a more consistent manner.

The finding that success rate was significantly higher when tactile feedback (P or B) was provided to the participants seems instead to contradict previous studies, where visual feedback outperformed tactile feedback [15], [16]. We argue that this apparent mismatch can be explained by the different protocols involved in the experiments. Both previous studies exploited a virtual environment, and one of them used a different control method (a Sensable Phantom [15]). In addition, subjects were told when the trial was starting and, consequently, when the object was going to slip. This allowed them to act by exploiting an internal model of the action built during previous trials. This is confirmed by the fact that the participants were able to perform the task, even if poorly, also without any feedback available (either visual or tactile). Although not directly tested, this was not possible for our subjects, because the event of hand opening (i.e. object slip) was unpredictable and fast (in the other studies the object was allowed to slip for a few seconds before the trial was marked as failed [16]). The present scenario was thus closer to what is experienced by upper limb amputees in real life.

The present study was indeed limited in some respects. Although very close to a real scenario, subjects were constantly listening to acoustic noise, the hand was mounted on a metal frame rather than attached to their arm and slippage was not caused by unwanted muscle activation (but triggered automatically using a PC). These choices were dictated by the need to mask the incidental feedback that the prosthesis could otherwise provide (e.g. motor noise and vibration) and to have repeatable experimental conditions. All these factors (lack of incidental feedback, presence of background noise and absence of concurrent EMG activation) could have affected the performance of the subjects [33]. However, this was a minor concern, as our goal was to compare the performance in the different feedback conditions rather than finding an absolute value.

Additionally, the subjects could not see the hand during both tactile feedback conditions, simulating the situation where the prosthesis is outside of the users' view. We did not test the condition where subjects were provided with both tactile and visual feedback, as in previous studies [13], [15]. We anyway anticipate that, in this case, the results would not change significantly. Indeed, humans integrate concurrent (multimodal) feedback in a way to minimize variance in the final task performance [34]. Thus, as tactile feedback was shown to outperform vision in all performance metrics, subjects would exploit mainly that source of information to perform the task successfully anyway.

Finally, we tested only unimpaired volunteers with little to no experience with myoelectric control. We argue that this probably did not affect the results significantly, as the 
myoelectric control scheme was very simple and all subjects readily mastered it. However, future studies with experienced myoelectric prosthesis users are needed in order to investigate how they perform under the different conditions tested, their confidence of handling objects with the prosthesis, and a possible change in cognitive burden during its use. It would also be interesting to understand whether patients with congenital limb deficiency would respond differently than amputees to the additional feedback or not. Indeed, these patients sometimes (10-20\%) report phantom sensations for the absent limb, suggesting that they may have an internal model of it [35]. We argue that major differences are not to be expected between these two populations, as the issue stated as the premise for our study is inherent to EMG control. Indeed, no particular differences in the ability of controlling a myoelectric prosthesis were reported among the two populations [36], [37]. However, more specific studies are needed to draw definitive conclusions.

\section{REFERENCES}

[1] R. S. Johansson and J. R. Flanagan, "Coding and use of tactile signals from the fingertips in object manipulation tasks," Nat. Rev. Neurosci., vol. 10, no. 5, pp. 345-359, 2009.

[2] R. Shadmehr, M. A. Smith, and J. W. Krakauer, "Error correction, sensory prediction, and adaptation in motor control," Annu. Rev. Neurosci., vol. 33, pp. 89-108, 2010.

[3] M. Kawato, "Internal models for motor control and trajectory planning,” Curr. Opin. Neurobiol., vol. 9, no. 6, pp. 718-727, 1999.

[4] R. S. Johansson and B. B. Edin, "Predictive feed-forward sensory control during grasping and manipulation in man," Biomed. Res. Tokyo, vol. 14, pp. 95-95, 1993.

[5] R. J. van Beers, "Motor Learning Is Optimally Tuned to the Properties of Motor Noise," Neuron, vol. 63, no. 3, pp. 406-417, 2009.

[6] R. F. ff. Weir and J. W. Sensinger, "Design of Artificial Arms and Hands for Prosthetic Applications," in Standard Handbook of Biomedical Engineering and Design, Second Ed., 2009, pp. 537598.

[7] P. A. Parker, K. B. Englehart, and B. Hudgins, "Myoelectric signal processing for control of powered limb prostheses," $J$.

Electromyogr. Kinesiol., vol. 16, no. 6, pp. 541-548, 2006.

[8] M. J. Ortiz-Catalan, R. Brånemark, B. Håkansson, and J. Delbeke, "On the viability of implantable electrodes for the natural control of artificial limbs: review and discussion," Biomed. Eng. Online, vol. 11, p. 33, 2012.

[9] E. A. Biddiss and T. T. Chau, "Upper limb prosthesis use and abandonment: a survey of the last 25 years," Prosthet. Orthot. Int., vol. 31, no. 3, pp. 236-257, 2007.

[10] R. Tomovic and G. Boni, "An adaptive artificial hand," IRE Trans. Autom. Control, vol. 7, no. 3, pp. 3-10, 1962.

[11] P. J. Kyberd et al., "MARCUS: A Two Degree of Freedom Hand Prosthesis with Hierarchical Grip Control," IEEE Trans. Rehabil. Eng., vol. 3, no. 1, pp. 70-76, 1995.

[12] C. Antfolk, M. D’Alonzo, B. Rosén, G. Lundborg, F. Sebelius, and C. Cipriani, "Sensory feedback in upper limb prosthetics," Expert Rev. Med. Devices, vol. 10, no. 1, pp. 45-54, 2013.

[13] D. D. Damian, A. H. Arita, H. Martinez, and R. Pfeifer, "Slip speed feedback for grip force control," IEEE Trans. Biomed. Eng., vol. 59, no. 8, pp. 2200-2210, 2012.

[14] C. E. Stepp and Y. Matsuoka, "Object manipulation improvements due to single session training outweigh the differences among stimulation sites during vibrotactile feedback," IEEE Trans. Neural Syst. Rehabil. Eng., vol. 19, no. 6, pp. 677-685, 2011.

[15] J. M. Walker, A. A. Blank, P. A. Shewokis, and M. K. O’Malley, "Tactile Feedback of Object Slip Facilitates Virtual Object Manipulation," IEEE Trans. Haptics, vol. 8, no. 4, pp. 454-466, 2015.

[16] H. Xu, D. Zhang, J. C. Huegel, W. Xu, and X. Zhu, "Effects of Different Tactile Feedback on Myoelectric Closed-Loop Control for
Grasping Based on Electrotactile Stimulation," IEEE Trans. Neural Syst. Rehabil. Eng., vol. 24, no. 8, pp. 827-836, 2016.

[17] R. S. Johansson and G. Westling, "Afferent signals during manipulative tasks in humans," in Information Processing in the Somatosensory System, London: Macmillan Education UK, 1991, pp. $25-48$.

[18] C. Cipriani, J. L. Segil, F. Clemente, R. F. ff. Weir, and B. B. Edin, "Humans can integrate feedback of discrete events in their sensorimotor control of a robotic hand," Exp. Brain Res., vol. 232, no. 11 , pp. 3421-3429, 2014.

[19] F. Clemente, M. D’Alonzo, M. Controzzi, B. B. Edin, and C. Cipriani, "Non-invasive, temporally discrete feedback of object contact and release improves grasp control of closed-loop myoelectric transradial prostheses," IEEE Trans. Neural Syst. Rehabil. Eng., vol. 24, no. 12, pp. 1314-1322, 2016.

[20] R. S. Johansson and G. Westling, "Signals in tactile afferents from the fingers eliciting adaptive motor responses during precision grip," Exp. Brain Res., vol. 66, pp. 141-154, 1987.

[21] D. A. Nowak, S. Glasauer, and J. Hermsdörfer, "How predictive is grip force control in the complete absence of somatosensory feedback?," Brain, vol. 127, no. 1, pp. 182-192, 2004.

[22] K. Kim and J. E. Colgate, "Haptic feedback enhances grip force control of sEMG-controlled prosthetic hands in targeted reinnervation amputees," IEEE Trans. Neural Syst. Rehabil. Eng., vol. 20, no. 6, pp. 798-805, 2012.

[23] N. Jorgovanovic, S. Dosen, D. J. Djozic, G. Krajoski, and D. Farina, "Virtual Grasping: Closed Loop Force Control Using Electrotactile Feedback," Comput. Math. Methods Med., vol. 2014, 2014.

[24] A. H. Bottomley, "Myoelectric Control of Powered Prostheses," Bone Joint J., vol. 47, no. 3, pp. 411-415, 1965.

[25] S. Weinstein, "Intensive and extensive aspects of tactile sensitivity as a function of body part, sex, and laterality," in The First International Symposium on the Skin Senses, 1968, pp. 195-222.

[26] G. Lundborg and B. Rosén, "Sensory relearning after nerve repair," Lancet, vol. 358, no. 9284, pp. 809-810, 2001.

[27] C. M. Galtrey, R. A. Asher, F. Nothias, and J. W. Fawcett, "Promoting plasticity in the spinal cord with chondroitinase improves functional recovery after peripheral nerve repair," Brain, vol. 130, no. 4, pp. 926-939, 2007.

[28] B. Rosén, G. Lundborg, L. B. Dahlin, J. Holmberg, and B. Karlson, "Nerve repair: correlation of restitution of functional sensibility with specific cognitive capacities," J. Hand Surg. Am., vol. 19, no. 4, pp. 452-458, 1994.

[29] R. Sigrist, G. Rauter, R. Riener, and P. Wolf, “Augmented visual, auditory, haptic, and multimodal feedback in motor learning: A review," Psychon. Bull. Rev., vol. 20, no. 1, pp. 21-53, 2013.

[30] G. H. Mowbray and M. V Rhoades, "On the reduction of choice reaction times with practice," $Q$. J. Exp. Psychol., vol. 11, no. 1, pp. 16-23, 1959.

[31] K. Kotani, S. Ito, T. Miura, and K. Horii, "Evaluating Tactile Sensitivity Adaptation by Measuring the Differential Threshold of Archers," J. Physiol. Anthropol., vol. 26, no. 2, pp. 143-148, 2007. B. Forster, C. Cavina-Pratesi, S. M. Aglioti, and G. Berlucchi, "Redundant target effect and intersensory facilitation from visualtactile interactions in simple reaction time," Exp. Brain Res., vol. 143, no. 4, pp. 480-487, 2002.

[33] M. Trimmel and G. Poelzl, "Impact of background noise on reaction time and brain DC potential changes of VDT-based spatial attention," Ergonomics, vol. 49, no. 2, pp. 202-208, 2006.

[34] M. O. Ernst and M. S. Banks, "Humans integrate visual and haptic information in a statistically optimal fashion," Nature, vol. 415, no. 6870, pp. 429-433, 2002.

[35] B. A. Philip, C. Buckon, S. Sienko, M. Aiona, S. Ross, and S. H. Frey, "Maturation and experience in action representation: Bilateral deficits in unilateral congenital amelia," Neuropsychologia, vol. 75, pp. 420-430, 2015.

[36] C. Cipriani et al., "Online myoelectric control of a dexterous hand prosthesis by transradial amputees," IEEE Trans. Neural Syst. Rehabil. Eng., vol. 19, no. 3, pp. 260-270, 2011.

[37] A. H. Al-Timemy, R. N. Khushaba, G. Bugmann, and J. Escudero, "Improving the Performance Against Force Variation of EMG Controlled Multifunctional Upper-Limb Prostheses for Transradial Amputees," IEEE Trans. Neural Syst. Rehabil. Eng., vol. 24, no. 6, pp. 650-661, 2016. 